\title{
MUJER Y COMUNICACIÓN EN EL FÚTBOL ESPAÑOL
}

\author{
María Jesús Fernández-Torres: Universidad de Málaga. España \\ mariajesusfernandez@uma.es
}

Ana Almansa-Martínez: Universidad de Málaga. España anaalmansa@uma.es

\section{RESUMEN}

El presente artículo intenta conocer el rol que las mujeres ejercen en un ámbito deportivo de gran calado como es el fútbol español. Para ello, el estudio se centra en su función dentro de los organigramas de los equipos de Primera y Segunda División así como en la investigación del clima de comunicación interna existente en dichas entidades deportivas. La gran importancia que posee este deporte, tanto por su capacidad de movilización de masas como por ser motor de inyección de ingentes cantidades de dinero, así como el papel que la mujer desempeña en la sociedad actual, justifican la elección de esta temática. La investigación se ha basado en el análisis cuantitativo y cualitativo de entrevistas y cuestionarios a más de cuarenta mujeres que trabajan dentro de los principales clubes de fútbol españoles profesionales. Los resultados del estudio no son muy esperanzadores pues la mujer aún hoy no forma parte del paisaje público de igual forma que lo hace el hombre.

PALABRAS CLAVE: Fútbol - Mujer - Comunicación - Estereotipos

\footnotetext{
${ }^{1}$ Autor correspondiente

María Jesús Fernández-Torres: Profesora Universidad de Málaga. Málaga. España

Correo: mariajesusfernandez@uma.es
} 


\title{
WOMAN AND COMMUNICATION IN SPANISH FOOTBALL
}

\begin{abstract}
This article tries to get to know the role of women in Spanish football. The reach has been focused on First and Second Division teams and the communication between the different clubs. The topic has been chosen because of the great importance of a sport that attracts millions of people and money, and we have also taken into account the importance of women in modern society. Our reseach has been based on interviews and questionnaires to more han forty women who work for the main Spanish professional football clubs. The results are not encouraging because women still do not have the same level of participation as men.
\end{abstract}

KEY WORDS: Football - Woman - Communication - Stereotypes

\section{INTRODUCCIÓN}

\subsection{El fútbol español}

Se hace necesario en este artículo contextualizar el campo sobre el que se realiza el estudio y dar unas breves pinceladas teóricas para conocer el terreno del que va a tratarse.

El fútbol (football) conocido también como balompié en España y "Soccer" en Estados Unidos, es un deporte que enfrenta a dos equipos formados por once jugadores cada uno (diez jugadores de campo y un portero o guardameta). El objetivo final es introducir con los pies el balón en la portería del equipo contrario.

El origen del fútbol tuvo lugar en Inglaterra. La Football Association desarrolló en 1863 gran parte de las reglas del juego que aún hoy rigen este deporte. En la actualidad, la Fedération Internationale de Football Association (FIFA) es el organismo rector del fútbol a nivel internacional. Este organismo, con sede en Zúrich (Suiza), realiza cada cuatro años la competición internacional más prestigiosa de este deporte rey, la Copa Mundial de la FIFA. 
Ciñéndonos al caso español, no hay que olvidar mencionar la Liga Nacional de Fútbol Profesional (LFP), asociación deportiva de derecho privado que a tenor de lo establecido en los artículo 12 y 41 de la Ley 10/1990 de 15 de Octubre del deporte², está integrada exclusiva y obligatoriamente por todas las Sociedades Anónimas Deportivas y Clubes que participan en competiciones oficiales de fútbol de ámbito estatal y de carácter profesional (Clubes de Primera y Segunda División). Es una asociación que carece de ánimo de lucro y es a ella a la que le corresponde legalmente la organización de dichas competiciones, en coordinación con la Real Federación Española de Fútbol (RFEF)³ , fundada en 1913.

La Liga Española de Fútbol es la principal competición entre equipos de fútbol de España que viene celebrándose de forma anual desde 1929, desarrollándose la temporada entre finales de agosto y/o principios de Septiembre y finales de Mayo y/o principios de Junio. El torneo se integra por un sistema de ligas interconectadas entre sí, cuya máxima categoría es la Primera División. Al término de cada temporada y en función de los resultados obtenidos, los equipos participantes pueden ascender o descender de división.

Tabla 1. El sistema de ligas se estructura de la siguiente manera

\begin{tabular}{|l|r|}
\hline \multicolumn{1}{|c|}{ CATEGORÍA } & NÚMERO DE EQUIPOS \\
\hline Primera División (Liga BBVA) & 20 equipos \\
\hline Segunda División (Liga Adelante) & 22 equipos \\
\hline Segunda División B & 4 grupos de 20 equipos cada uno $Æ$ 80 \\
\hline Tercera División & 18 grupos de 20 equipos cada uno $Æ$ \\
& \\
\hline Divisiones Regionales & El número varía según la Comunidad. \\
\hline
\end{tabular}

Fuente: Elaboración propia

\footnotetext{
2 Publicado en BOE número 249 de 17/10/1990, páginas 30397 a 30411 (15 páginas).

3 La Real Federación Española de Fútbol está afiliada, desde 1914, a la FIFA, que gobierna las federaciones de fútbol a nivel mundial; desde 1954, a la UEFA (Union of European Football Associations), que es la confederación de asociaciones nacionales de fútbol y máximo ente de este

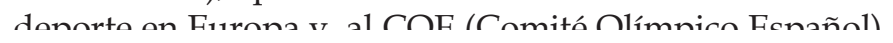




\section{METODOLOGÍA}

Las reflexiones y resultados que aquí se exponen pretenden contribuir al desarrollo de los estudios sobre género y estereotipos para permitir establecer el tratamiento que el deporte, más concretamente el fútbol, hace de ambos géneros $\mathrm{y}$, el papel que los hombres y mujeres desempeñan dentro de clubes que se han convertido en empresas.

La gran importancia que este deporte tiene, no sólo por capacidad de movilización de masas sino también por inyectar ingentes cantidades de dinero, así como los roles que la mujer desempeña en la sociedad actual, justifican la elección de esta temática de estudio.

Para realizar esta investigación he elaborado un análisis cuantitativo y cualitativo, señalando como principal objetivo de la misma conocer el rol de la mujer dentro de los clubes de fútbol profesionales de nuestro país así como el clima de comunicación interna existente en tales entidades deportivas.

La metodología utilizada se ha basado en la confección de cuestionarios y entrevistas a mujeres que trabajan en diferentes ámbitos de los clubes de fútbol: Secretarias de Presidencia, Prensa, Relaciones Externas, Administración, Taquillas, etc.

La muestra es fruto de la selección aleatoria de clubes de fútbol profesionales españoles así como de otros entes futbolísticos, y de mujeres que trabajan en los mismos. Fueron más de cien las encuestas enviadas, de las cuales fueron contestadas treinta. La muestra abarca a trabajadoras de los clubes profesionales de la Liga Española entre los que cabe destacar: Real Madrid C.F., Club Atlético de Madrid, Valencia C.F., Sevilla F.C., Málaga C.F., Cádiz C.F., Unión Deportiva Las Palmas, ..., entre otros, así como entidades deportivas como la Liga de Fútbol Profesional o la Real Federación Española de Fútbol.

Asimismo, se ha llevado a cabo una búsqueda exhaustiva de información de la temática que aquí se trata para dotar de un marco teórico a la misma.

La investigación se estructura en torno a cuatro bloques bien diferenciados, dividiéndose el cuestionario en cuatro partes, con un total de treinta y nueve preguntas:

a) En la primera de ellas, se pregunta tanto el club de fútbol al que pertenece la mujer encuestada como categoría, franja de edad, nivel de estudios, cargo en la empresa y departamento al que se adscribe y años que lleva en la empresa. 
b) En la segunda parte, y con una batería de ocho preguntas, se intenta profundizar en el conocimiento de las trabajadoras respecto de la filosofía del club y de su cultura empresarial: "¿Conoce el año de creación de su empresa?, ¿Sabe si ésta posee alguna postura preestablecida respecto a la sociedad, etc.".

c) La tercera parte, compuesta por diecisiete preguntas, se centra en la satisfacción con el trabajo desempeñado así como el clima de comunicación interna existente en la entidad, tanto a nivel global como con diferentes estamentos de la misma en igualdad de condiciones profesionales y retributivas, ¿se cambiaría a otra empresa para desarrollar un trabajo similar?, ¿qué opinión le merece el clima interno que existe en su empresa en cuanto a promoción interna, comunicación con superiores, compañerismo, ...?, ¿cómo considera su nivel de formación para el desempeño de su trabajo?, herramientas de comunicación interna, etc.

d) En la última parte, con un total de diez preguntas, se pretende dilucidar si el fútbol es un mundo de hombres, no en lo que a afición se refiere, sino a la hora del trabajo interno que se desempeña en los mismos: "¿Su superior es hombre o mujer?, ¿Cuántas mujeres y hombres trabajan en el equipo directivo?, ¿el trato por ser mujer es diferente?, etc.".

\section{ANÁLISIS Y DISCUSIÓN}

\subsection{La comunicación en fútbol}

La profesionalización del fútbol se ha incrementado enormemente en los últimos años haciendo que su gestión haya ido tomando mayor importancia. Por una parte, la venta de los derechos de televisión y, por otra, el desarrollo del merchandaising han ampliado no sólo las actividades de los clubes sino también, sus ingresos.

El fútbol por tanto es más que un deporte, enmarcándose en un clima en el que se mueven ingentes cantidades de dinero y en donde el tiempo y el espacio diario dedicado en medios a este "deporte rey" es cada vez más significativo. Todo ello sin olvidar el enorme número de aficionados que posee, siendo conocido como el negocio del deporte (Chadwick \& Beech, 2004) o la industria del deporte (Foster et al., 2006) por ser uno de los más populares, que levanta pasiones y mueve masas.

Ello exige no sólo la profesionalización de los considerados como sus principales actores (los futbolistas) sino también de los propios clubes que necesitan profesionales preparados adecuadamente con el objetivo último de conseguir mayor eficacia y eficiencia en la gestión. 
Según el Deloitte Football Money League 2011, este es el Top 20 de los ingresos de clubes en millones de euros:

Tabla 2. Top 20 de los ingresos de clubes en millones de euros

\begin{tabular}{|c|c|c|}
\hline Position & Club & $2009 / 2010$ revenue $(€ \mathrm{~m})$ \\
\hline 1 & Real Madrid & 438,6 \\
\hline 2 & FC Barcelona & 398,1 \\
\hline 3 & Manchester United & 349,8 \\
\hline 4 & Bayer Munich & 323,0 \\
\hline 5 & Arsenal & 274,1 \\
\hline 6 & Chelsea & 255,9 \\
\hline 7 & AC Milan & 235,8 \\
\hline 8 & Liverpool & 225,3 \\
\hline 9 & Internazionales & 224,8 \\
\hline 10 & Juventus & 205,0 \\
\hline 11 & Manchester City & 152,8 \\
\hline 12 & Tottenham Hotspur & 146,3 \\
\hline 13 & Hamburguer SV & 146,2 \\
\hline 14 & Olympique Lyonnais & 146,1 \\
\hline
\end{tabular}




\begin{tabular}{|l|r|r|}
\hline 15 & Olympique Marseille & $\mathbf{1 4 1 , \mathbf { 1 }}$ \\
\hline 16 & Schalke 04 & $\mathbf{1 3 9 , 8}$ \\
\hline 17 & Atlético de Madrid & $\mathbf{1 2 4 , 5}$ \\
\hline 18 & AS Roma & $\mathbf{1 2 2 , 7}$ \\
\hline 19 & VfB Stuttgart & $\mathbf{1 1 4 , 8}$ \\
\hline 20 & Aston Villa & $\mathbf{1 0 9 , 4}$ \\
\hline
\end{tabular}

Fuente: Deloitte Football Money League 2011

Entre estos 20 equipos, tres son españoles: dos de ellos ocupan el primer y segundo puesto (Real Madrid, con unos ingresos de 438,6 millones de euros y FC Barcelona, con 398,1 millones de euros). El tercer equipo español es el Atlético de Madrid, situado en el puesto número 17 , con 124,5 millones de euros.

Por todo ello, un club profesional deja de ser una organización exclusivamente deportiva para ser, a su vez, una empresa. Consecuentemente, su gestión obliga a darle un enfoque empresarial y productivo. Además del resultado, es necesario conseguir una cuenta de resultados saneada, solvente, rentable, eso sí, sin dejar atrás el conseguir una afición fiel, comprometida con la entidad y la materialización de los valores deportivos.

Al convertirse en una empresa, cuenta con las mismas obligaciones y problemas, estos últimos causados a menudo por la falta de comunicación que existe dentro de su organización. Pero hablar de fútbol y comunicación es complicado pues, desmenuzar los códigos dentro de un club, es tarea ardua y difícil porque guardan celosamente sus estrategias ya que no quieren que sus oponentes conozcan sus códigos, formas de trabajar, trato con los jugadores...

Harris y Harris (1987), hablan de la comunicación que debe existir entre entrenador y equipo y los objetivos que debe tener: persuadir, evaluar, informar, motivar y resolver problemas. Estrategias encauzadas a alcanzar un feedback eficiente; estrategias que, desde mi punto de vista, pueden extrapolarse a todos los públicos, internos y externos, de una entidad futbolística, es decir: jugadores, propietarios, trabajadores, socios, aficionados, empresas, ciudad y medios de comunicación. 
Muchos teóricos consideran que 1992 supuso para el deporte español un profundo cambio no sólo por los Juegos Olímpicos en Barcelona sino porque el nuevo marco jurídico del deporte profesional, hizo que los clubes de fútbol y baloncesto se transformasen en sociedades anónimas deportivas. Ello trajo aparejado grandes renovaciones organizativas en las estructuras de los equipos deportivos y en una de las áreas, la de comunicación.

Se desarrolla la figura del jefe de prensa que pasa a ser un elemento claro para fijar estrategias comunicativas de propaganda y crisis, proyectar imagen en la sociedad, mejorar su relación con los públicos y crear un clima favorable de opinión al consumo de los productos que comercializan los clubes. (Castañón Rodríguez, 2008) ${ }^{4}$. Asimismo, destaca que la labor de los Gabinetes de Comunicación en el mundo del deporte se centran en: enlazar la identidad local y nacional, coordinar el cambio de consumo generado por la televisión y convertir las nuevas tecnologías en un espacio de comunicación entre profesionales, periodistas y aficionados.

Los medios de comunicación son verdaderos creadores de opinión publicando un volumen considerable de información futbolística. Debido a ello, mantener un clima cordial con ellos resulta esencial para conseguir un buen posicionamiento mediático que ayude a su vez a captar nuevos socios y aficionados. Pero, para lograr este objetivo, es necesario contar en los clubes con un ámbito específico encargado exclusivamente del área comunicativa y utilizar todas las herramientas al servicio de la comunicación para lograr, a través de los medios, acercarse a su público potencial, transmitir una buena imagen de la entidad y ser parte diaria de la agenda-setting de los medios.

\subsection{Fútbol y estereotipos}

Probablemente, no haya algo que defina de una forma tan concluyente la sociedad en la que vivimos respecto a los roles de género y los estereotipos que el hablar de fútbol. A través de los siglos, la mirada en el deporte se ha construido desde el punto de vista del varón siendo el fútbol considerado, en la mayoría de los casos, y tal y como señala Archetti (1985), un ethos masculino.

Es evidente que el discurso futbolístico proviene, en su mayoría, del universo masculino y por ende, las reglas y los valores que circulan por su práctica pertenecen a su dominio. Por tanto, ¿qué ocurre cuando la mujer se introduce en este mundo masculino?.

${ }^{4}$ En su reseña de la tesis de grado "The press office of the Real Sporting of Gijón. Communication in

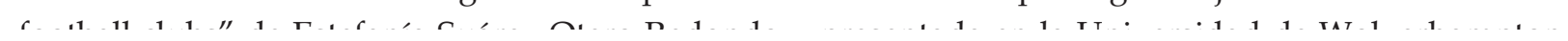


Preguntas como las que se formulan a continuación, arrojan respuestas con cifras muy dispares y diferentes y quizá por ello, muchos se escuden en argumentar que el fútbol es hoy por hoy un deporte exclusivo de hombres: ¿Cuántos hombres juegan al fútbol?, ¿Cuántas mujeres juegan?; ¿Cuántos hombres asisten a los estadios?, ¿Cuántas mujeres lo hacen?; ¿Cuántos hombres ven el fútbol en televisión, lo escuchan en radio o leen prensa deportiva?, ¿Cuántas mujeres?; etc. Esta particular Ley de Oferta y Demanda refleja una visión simplista del género dentro de este "deporte rey". Porque entonces, ¿no tenemos en cuenta las cifras que arrojan las mujeres?. En una sociedad a la que se le atribuye el adjetivo de "igualitaria", se deben romper los anacronismos e ir derribando barreras y estereotipos.

Lippman ${ }^{5}$ (1922) fue uno de los primeros en definir el vocablo "estereotipo" y lo conceptualizó como "molde". Posteriormente, definiciones más recientes delimitan el estereotipo a una imagen construida sobre un grupo de gente que resulta de la selección de unos pocos símbolos entre un elevado número de posibilidades para representar al grupo. El estereotipo no entiende de individualidades; si se pertenece a un grupo se es definido en función a la simplificación que se haga del mismo. La mujer, por el hecho de pertenecer al grupo estereotipado, es definida como tal.

A la mujer se la estereotipa como manipuladora, seductora, harpía, dependiente, maternal, sensible, con el rol tradicional de ama de casa, ...; en cambio, al hombre, se lo estereotipa como héroe, inteligente, conquistador, valiente, leal, cabal, ...

Por tanto, dos son las características básicas de los estereotipos: la representación simplificada de la realidad y la resistencia al cambio. Por otro lado, y volviendo al tema que nos ocupa, resulta difícil concebir el fútbol sin el uso de estereotipos y clichés.

Los estereotipos son injustos porque no son exactos y tienen efectos negativos sobre el grupo estereotipado. Quin y Barrie (. Quin \& Barrie 1997), hablan de los

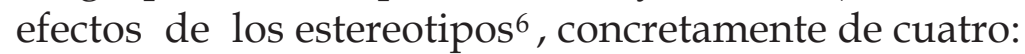

a) La justificación de nuestras actitudes y creencias que parecen normales en una sociedad sexista: la mujer debe dejar de trabajar cuando tiene un bebé, ... Creemos que lo común es lo normal y lo correcto.

b) La estabilidad del sistema: Las personas estereotipadas contarán con menos oportunidades.

\footnotetext{
${ }^{5}$ Walter Lippman, en su libro "Public Opinion", cuestiona que sea posible una democracia real en la sociedad moderna y fundamenta esta crítica argumentando, entre otros, que los individuos no son observadores objetivos, sino que sus estereotipos e intereses hacen percibir la realidad de modo diverso y limitado. en su noción de estereotipo.

${ }^{6}$ En su libro "Historias y estereotipos", los autores se centran en analizar el triunfo en la actual sociedad mediática de estereotipos sociales, sexuales, económicos, ... y que son calificados como "positivos" y "negativos".
} 
c) Discriminación: Las mujeres cobran menos que los hombres ejerciendo el mismo trabajo; las mujeres apenas pueden acceder a puestos de responsabilidad altamente remunerados, ...

d) Falta de pensamiento crítico y de conocimiento.

Se suele pensar que los estereotipos cambian porque cambian los símbolos con los que se representan, pero los valores asociados siguen permaneciendo.

La mujer constituye un grupo desempoderado respecto al género masculino y ello lleva a una continua necesidad de aprobación que las mujeres tienen en sus culturas y en los ámbitos en donde ejercen su actuación.

La participación de la mujer en el deporte es menor que la del hombre y su acceso al mundo deportivo ha sido tardío y ha estado sembrado de dificultades ya que continuamente han tenido que ir superando barreras creadas por infinidad de estereotipos tanto sociales como culturales. Dificultades que han ido venciendo y que, en parte, tienen su origen en la propia gestación del deporte que fue ideado, por y para hombres, como vía de transmisión de determinados valores y para el desarrollo de sus capacidades físicas.

Así, Pierre de Coubertin7 , argumentaba que la presencia de la mujer en un estadio resultaba antiestética, poco interesante e incorrecta.

De hecho, si analizamos la imagen de mujeres en los estadios podemos dar cuenta de la mirada masculina que recae sobre ella y que va encaminada, fundamentalmente, a dos tipos de féminas: por una parte, la tradicional mujer doméstica (madre, esposa, hija) o, por otra, la mujer sensual.

Es por ello que resulta necesario definir su identidad en este particular universo más allá de constituirla de forma heterónoma, con los valores y las reglas del otro.

\subsection{Fútbol y mujer}

Con todo lo dicho hasta el momento, resulta lógico abogar por romper las barreras en el deporte $y$, particularmente, en fútbol, ya que la sociedad sigue siendo machista en este campo. Hay que buscar la cualificación para desempeñar el trabajo, independientemente del género de la persona.

Según un estudio realizado por la consultora de Recursos Humanos Hudson en Diciembre de 2010, el número de mujeres directivas aumentó un 3\% en los últimos 5 años, aunque las féminas sólo representan el 26,9\% del total de trabajadores con este rango. El análisis fue elaborado a partir de 72.120 directivas de entre 30 y 60 años y, a su vez, apunta que el porcentaje de mujeres es más significativo en los mandos 
intermedios. Existen sectores como el digital, el industrial o el de finanzas, donde predomina claramente el perfil masculino. El estudio también muestra que el número de mujeres directivas se equipara al de hombres en sectores como Recursos Humanos, Marketing, Calidad y Atención al Cliente.

Los resultados de este estudio son perfectamente extrapolables al mundo de los clubes de fútbol ya que, aunque la mujer poco a poco ha ido integrándose en todos los ámbitos de la sociedad, aún existen sectores en los que su integración resulta bastante limitada. Esto es lo que ocurre en fútbol, donde ver a mujeres directivas no es habitual y aún menos si el cargo es de Presidenta.

Los medios dedican un minuto al deporte femenino por cada 19 minutos al masculino e incluyen una noticia de deporte femenino para cada 15 minutos del masculino. Este dato ${ }^{8}$ es otro reflejo más de la importancia de sensibilizar a la audiencia, a periodistas y a deportistas en su conjunto hacia el uso de un lenguaje no sexista y la eliminación de la influencia de estereotipos en la información.

Tal desigualdad en el trato comienza en las directivas de los equipos, donde hay menos puestos para las mujeres, que en muy pocos casos realizan gestiones relevantes en los clubes.

Se hace, pues, necesario un pacto social de género que redefina las funciones de mujeres y hombres en la sociedad. La ayuda institucional que se otorga a los clubes femeninos es escasa así como los bajos sueldos que, por lo general, tienen las deportistas, sueldos que no les permiten exclusivamente vivir del deporte. De hecho, desde muchos cargos deportivos, se aconseja a las deportistas estudiar, para poder compatibilizar su profesión y su estudio con los entrenamientos y los partidos.

El giro que se está produciendo en el deporte rey no puede dejar inerme a la sociedad puesto que el fútbol es mucho más que fútbol, es un hecho social. Últimamente está muy en boga la importancia de los valores en este deporte: la necesidad de transmitir afecto al jugador, trabajo en equipo, aprender de la derrota, humildad, compañerismo, afán de superación, ... Pero esta importancia no lleva acompañado un cambio en los protagonistas.

\footnotetext{
${ }^{8}$ Dato extraído de la Charla-Coloquio "Tomando el pulso al deporte femenino en la Comunidad de Madrid", puesta en marcha a iniciativa de Deporte Femenino Visible y que tuvo lugar en Coslada (Madrid) en Abril de 2011.
} 
El triunfo de esos valores deportivos no lleva aparejado el que existan más mujeres en los equipos técnicos, el que existan más jugadoras o más mujeres en las directivas de los clubes. Por ello, es importantísimo que este deporte evolucione con el tiempo y se modifiquen las políticas deportivas para el desarrollo de estrategias que fomenten la presencia y el mantenimiento de técnicas, jugadoras y directivas.

\section{CONCLUSIONES}

El análisis pormenorizado de cada uno de los cuestionarios me ha llevado a inferir las conclusiones que, a posteriori, detallo. He de señalar la dificultad para obtener las respuestas al mismo, siendo muchas mujeres reacias a realizarlo por temor a que éste pudiera perjudicarlas laboralmente. De hecho, hubo un amplio colectivo que no permitió que se le hiciera y de los realizados, unos, han tenido que descartarse por ser contradictorias las respuestas entre sí y, otros, han contado con algunas preguntas sin contestar.

El perfil de las trabajadoras que han participado en la muestra es el siguiente:

Todas ellas pertenecen a clubes de fútbol profesional (tanto de Primera como de Segunda División). El 55\% cuenta con estudios universitarios, el 10\% con estudios de formación en Grado Superior y el 35\% con estudios medios. Todas las encuestadas tienen una media de edad comprendida entre los 30 y los 50 años y su tiempo en la entidad a la que pertenecen es considerable, siendo la media de la muestra de 9 años. Asimismo, las labores que realizan se centran en áreas administrativas (40\%), Marketing (20\%), el 25\% son trabajadoras de más alto rango (sin llegar a ser directivas) y el 15\% restante se dedica a otras actividades como instalaciones, taquillas...

La segunda parte del cuestionario iba destinada a averiguar el conocimiento de las trabajadoras sobre la filosofía de la entidad y la cultura empresarial. Los datos arrojan las siguientes cifras:

El 95\% conoce el año de creación de su empresa y el 80\% conoce bien la misión de la entidad para la que trabaja, es decir, sus metas, propósitos... Asimismo, un $85 \%$ sabe con certeza a quién pertenece la entidad y sólo dudan de ello, un 15\%. Ocurre lo mismo con el organigrama empresarial, lo conocen muy bien el $85 \%$ de las encuestadas, mientras que el $20 \%$ lo conoce en parte o dudan de él. Respecto a si recuerdan o no crisis en su empresa, el 65\% sí lo hace, mientras que el 35\% restante no la recuerda o sólo ha escuchado hablar de alguna en contadas ocasiones. 
Una mayoría, el $65 \%$, considera que su club se posiciona en relación con los demás cerca del líder o incluso es líder y un 35\% considera que se encuentran alejados del líder del sector. Otra de las preguntas más representativas de esta parte, es el grado de identificación e interiorización con el club en el que trabajan. El $70 \%$ se siente bastante o muy identificada con él, mientras que el 30\% restante se siente poco o nada identificada.

El tercer bloque, uno de los más extensos, se centra en la satisfacción de las trabajadoras con su trabajo así como en el clima interno de la entidad y la comunicación en la misma. Un 55\% de las encuestadas consideran su nivel de formación profesional muy superior a las necesidades del puesto que desempeñan mientras que el $45 \%$ restante cree que su formación es acorde a la actividad que desempeña. Asimismo, el 50\% considera su tarea profesional compleja y de alta responsabilidad.

En general, el $90 \%$ de ellas está bastante satisfecho con su trabajo frente a un 10\% que no lo está. Sin embargo, en igualdad de condiciones profesionales y retributivas, un $25 \%$ se cambiaría a otra empresa para desarrollar un trabajo similar y dudan de ello un $45 \%$. Sólo un 30\% permanecería en la entidad. Respecto al salario recibido, hay clara división de opiniones, un 50\% está bastante satisfecho con su remuneración, mientras que el otro $50 \%$ no lo está.

Las relaciones con sus superiores también han sido estudiadas y merece la pena destacar que el 85\% afirma tener muy buenas relaciones con ellos y el 65\% se sienten plenamente apoyadas en el desarrollo de su trabajo. Son estas mismas trabajadoras las que definen esta relación como de estrecha colaboración, confianza y respeto mutuo. En cambio, el 15\% destaca que las relaciones con sus superiores no son favorables y un $35 \%$ no se sienten apoyadas por ellos en su trabajo. Éstas definen su relación con los superiores con poca confianza e incluso obstrucción.

Respecto a si la dirección se encuentra, desde su punto de vista, alejada o no de lo que ocurre en la entidad, el $70 \%$ cree que sí, frente a un $30 \%$ que considera lo contrario.

Respecto al clima interdepartamental el $40 \%$ señala que los departamentos de las entidades cooperan satisfactoriamente entre sí, frente al $60 \%$ que destaca que colaboran poco o nada.

Asimismo, también se les preguntó sobre la imagen que el club ofrece al exterior, siendo positiva para el $70 \%$. Sobre el clima interno existente en las entidades de fútbol, los cuestionarios arrojan los siguientes datos:

a) El 85\% considera que la promoción interna es inexistente y mala.

b) El $80 \%$ considera que es buena la relación que mantienen con sus 
c) El $65 \%$ mantiene que la colaboración laboral es buena frente al $35 \%$ que cree todo lo contrario.

d) El $75 \%$ señala que tiene libertad individual frente a un $20 \%$ que se encuentran más coaccionadas en el desempeño de sus funciones.

Asimismo, el 75\% señala que la empresa las mantiene informadas sobre movimientos de personal, planes... frente a un $25 \%$ que apunta no recibir información al respecto.

Destacar que todas las entidades cuentan con departamento de comunicación y que las herramientas más empleadas de Comunicación Interna son los correos electrónicos, las cartas a socios y circulares.

El último apartado del cuestionario pretende dilucidar no sólo los datos respecto a género en un club de fútbol, sino también, la opinión que como trabajadoras tienen sobre la visión del fútbol como un deporte de hombres, no sólo en lo que a afición se refiere, sino también a la hora de trabajar en los clubes. Los datos son los siguientes:

a) El 95\% de sus superiores son hombres. Sólo un 5\% dice tener como superior a una mujer.

b) La mitad de la muestra considera que el fútbol es un mundo de hombre y, la otra mitad, lo contrario. En esta respuesta cabe destacar el hecho de que ellas mismas no entienden que esto sea así o no lo quieren entender y abogan por la defensa de que el fútbol es también un deporte para mujeres.

c) También existe equilibro al considerar si el ambiente interno de un club es"machista" o no. El 50\% opina que bastante y el otro $50 \%$ considera que poco.

d) El 55\% no tiene la sensación de ser una intrusa en su dinámica diaria de trabajo, frente a un $45 \%$ que lo tiene en ocasiones o de forma continua.

e) El 50\% refleja que el trato por ser mujer dentro de la empresa no es diferente, frente al otro $50 \%$, que cree que sí lo es y dentro de este último porcentaje, un

f) $40 \%$ cree que el trato por ser mujer es peor.

g) Un $45 \%$ considera que el trabajo que desempeña en el club le ha sido asignado por ser mujer y que podría estar desempeñando otro si fuese hombre. En cambio, un 55\% considera que su trabajo no se lo han otorgado por su género.

h) Un $70 \%$ de las trabajadoras señalan que su círculo social se sorprende cuando conocen que trabajan en un club de fútbol.

i) Apuntan que el ámbito directivo está compuesto en su inmensa mayoría por hombres y que son éstos los que más personas poseen a su cargo. 
Así pues, la mayor parte de las mujeres encuestadas, aunque en su mayoría cuentan con estudios superiores, realizan funciones centradas en tareas administrativas y ocupan puestos de no muy alta responsabilidad. Asimismo, una inmensa mayoría conoce perfectamente la filosofía del club en el que trabajan y la identificación con el mismo es plena. En ello, puede tener que ver, el hecho de que la relación con sus superiores y con los compañeros sea, en la mayor parte de los casos, satisfactoria.

Los clubes deberían centrar su mirada en varios aspectos internos. Cuentan con mujeres que realizan tareas teniendo un nivel de formación superior a las necesidades del puesto. Asimismo, el estudio arroja datos que reflejan que las directivas se encuentran alejadas de lo que acontece en la entidad y que la colaboración entre los distintos departamentos es escasa, con las consiguientes consecuencias. Otro factor negativo es la poca o nula capacidad de promoción interna que hay entre las trabajadoras.

Además y en lo que respecta a género, las cifras hablan por sí solas. Los superiores en una inmensa mayoría, son hombres y el ámbito directivo no cuenta prácticamente con ninguna mujer o, si las hay, son pocas.

La única pregunta abierta que se insertó en el cuestionario informa de la opinión de muchas mujeres sobre la causa por la que creen que no existen más féminas en el fútbol. Hay un consenso general en definir a este "deporte rey" como "un mundo de hombres" o "mundo machista", si bien hay algunas voces femeninas que se alzan diciendo que no existen más mujeres porque se requiere mucho tiempo y dedicación al mismo y que el fútbol es lo que es por futbolistas, que son hombres.

Esto último hace replantearse si algunas mujeres, en alguna ocasión, fomentan el "machismo". Otras consideran que los hombres no admiten que la mujer pueda realizar labores dentro del club de una forma mejor a la que lo hacen ellos. Asimismo, señalan a la sociedad como la culpable de esa visión y ello lo avala el hecho de que un porcentaje muy alto de su círculo social ve con perplejidad que estas mujeres desempeñen su labor en este campo.

Para concluir, decir que la mujer aún hoy no forma parte del paisaje público de igual forma que lo hace el hombre. Nos guste o no, aún somos una "sociedad machista" y ello se refleja en este deporte. Quizá habría que cambiar la educación, ganar en respeto y aprender del fútbol femenino, un deporte más democrático y más modesto que el masculino. ¿Se romperá alguna vez un tabú que por ahora parece casi una aspiración utópica? Esperemos que así sea. 


\section{REFERENCIAS}

Agudo San Emeterio, Á. (2003). Marketing del fútbol. Madrid: Pirámide.

Ambrosiano, M. (2006). El gran libro del fútbol. Madrid: Ediciones Paulinas.

Archetti, E. (1985). Fútbol y ethos. Monografías e Informes de Investigación, 7.

Battle, R. et al. (2011): The Untouchables Football Money League. Manchester: Sports Business Group at Deloitte.

Castañón Rodríguez, J. (2008). Lenguaje y comunicación en el Real Sporting de Gijón, SAD. Revista Digital, Buenos Aires, 12(117). Recuperado el 28 de Julio de 2011, de http://www.efdeportes.com/efd117/lenguaje-y-comunicacion-en-el-real-sportingde-gijon.htm

Chadwick, S. \& BEECH, J. (2004). The Business of Sport Management. Harlow: Prentice Hall.

Foster, G., Greyser, S. A. \& Walsh, B. (2006). The Business of Sports: Text and cases on strategy and Management. South-Western: Thomson.

Galeano, E. (2006). El fútbol a sol y sombra. Madrid: Siglo XXI.

Harris, D.V \& Harris, B.L. (1987). Psicología del deporte. Integración mente-cuerpo. Barcelona: Hispano Europea.

Lippmann, W. (2003). La opinión pública. Madrid: S.L. Cuadernos de Langre.

López Díez, P. (2004). Manual de información en género. Madrid: Instituto RTVE.

Mcmahon, B. \& Quin, R. (1997). Historias y estereotipos. Madrid: Ediciones De La Torre.

Toft, T. (2003). TV Rights of sport events. Bruselas: Comision Europea.

\section{María Jesús Fernández Torres}

Doctora en Ciencias de la Comunicación por la Universidad de Málaga en 2004, con una tesis que versó sobre Movimientos Sociales y estrategias de comunicación en las ONG. Fue, en su momento, la Doctora más joven de España. Técnico Auxiliar en Protocolo. Especializada en Comunicación Interna y Externa. Funcionaria de carrera y profesora de la Universidad de Málaga. 


\section{Ana Almansa Martínez}

Profesora en el Departamento Comunicación Audiovisual y Publicidad, en la facultad de Ciencias de la Comunicación de la Universidad de Málaga, donde imparte las asignaturas, Comunicación Politica, Técnicas y Herramientas de las Relaciones Públicas y Aportaciones de las Nuevas Tecnologías a la Gestión de la Comunicación Externa. Asimismo, es docente en postgrados de la Universidad de Almería, de la Universidad Mayor de Chile y de la Universidad de Málaga. Universidad de Málaga; y directora académica de los Cursos de Especialización Universitaria Comunicación para la Igualdad. Su línea de investigación es la comunicación institucional, empresarial y organizacional y la incidencia que en estos ámbitos están teniendo las nuevas tecnologías. 